\section{Pancreatic-colonic fistula successfully treated with endoscopic transpapillary nasopancreatic drainage}

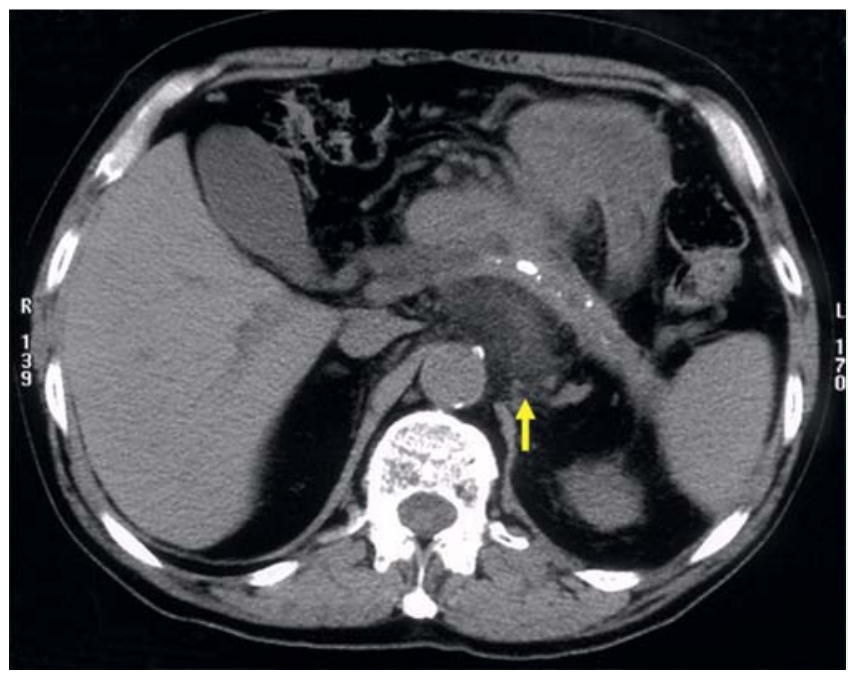

Fig. 1 Abdominal computed tomography (CT) scan showing atrophy and calcification of the pancreas and fluid collection in the anterior pararenal space (arrow).
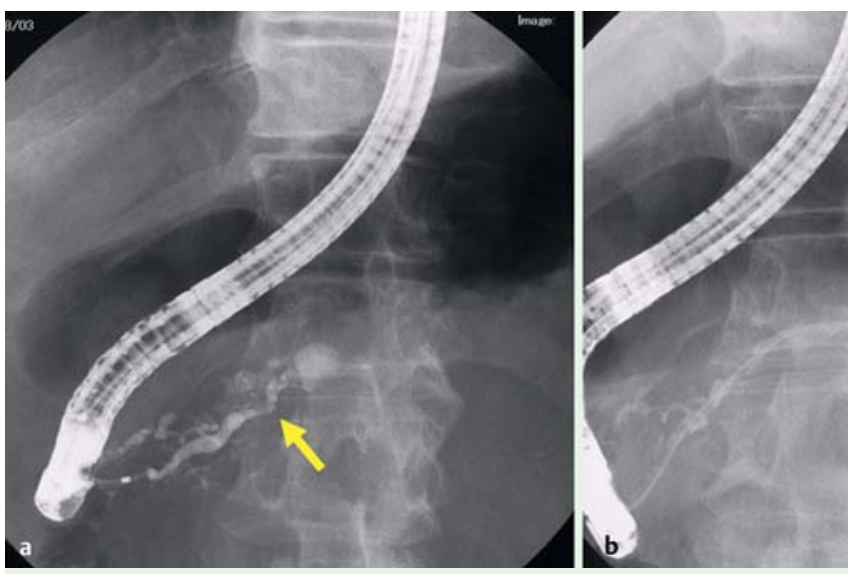

Fig. 2 a, b Endoscopic retrograde pancreatography showing irregular stenosis of the main pancreatic duct (arrow) and fluid collection in the tail of the pancreas (arrowhead).

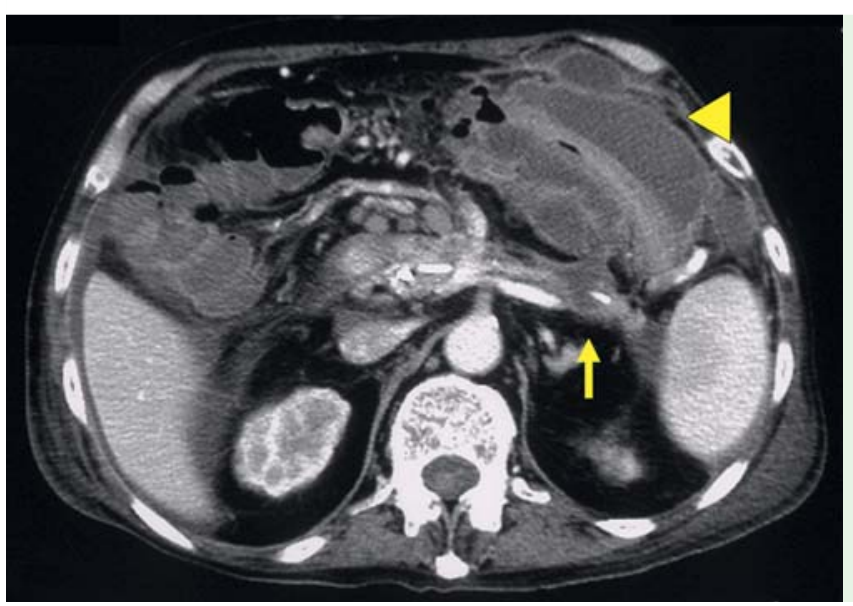

Fig. 3 Computed tomography (CT) scan showing dilatation of the distal main pancreatic duct (arrow) and inflammation around the transverse colon (arrowhead).
A 70-year-old man with upper abdominal pain was diagnosed as having an acute exacerbation of chronic pancreatitis. He was a heavy drinker, with a history of severe acute pancreatitis at 68 years of age. Abdominal computed tomography (CT) showed atrophy and calcification of the pancreas and fluid collection in the anterior pararenal space ( $\bullet$ Fig. 1 ). Initial treatment comprised total parenteral nutrition and antibiotics, which led to improvement in symptoms and laboratory data. However, the pancreatitis relapsed after food intake was started. Endoscopic retrograde pancreatography (ERP) showed irregular stenosis of the main pancreatic duct (MPD) and fluid collection in the tail of the pancreas ( $\bullet$ Fig. 2). Hence, endoscopic pancreatic stent drainage was carried out. After 2 weeks, the patient developed fever accompanied with mucobloody stool. However, the bleeding point was not detected on endoscopy. CT showed dilatation of the distal MPD and inflammation around the transverse colon ( Fig. 3). The muco-bloody stool improved gradually with total parenteral nutrition and blood transfusion, in addition to antibiotic treatment. ERP after another 2 weeks revealed a colonic fistula at the distal MPD ( Fig. 4 a). Therefore, an endoscopic transpapillary nasopancreatic drainage (ENPD) tube was placed in the distal MPD. After 10 days, there were no signs of the fistula at scanning with a contrast medium ( Fig. 4 b), and the inflammation around the transverse colon had resolved ( $\bullet$ Fig. 5 ).

Pancreatic-colonic fistula is an uncommon but potentially lethal complication of severe acute pancreatitis. Because of its frequent association with sepsis or bleeding, appropriate operative intervention is necessary [1,2]. Although successful endoscopic interventions such as endoscopic pancreatic stent or transgastric nasocystic drainage catheter placements have been reported [3-5], the successful use of transpapillary nasopancreatic drainage alone has not been reported previously. Here, we present a case of a pancreatic-colonic fistula associated with pancreatitis that was successfully treated with ENPD.

Endoscopy_UCTN_Code_TTT_1AR_2AK

Competing interests: None 

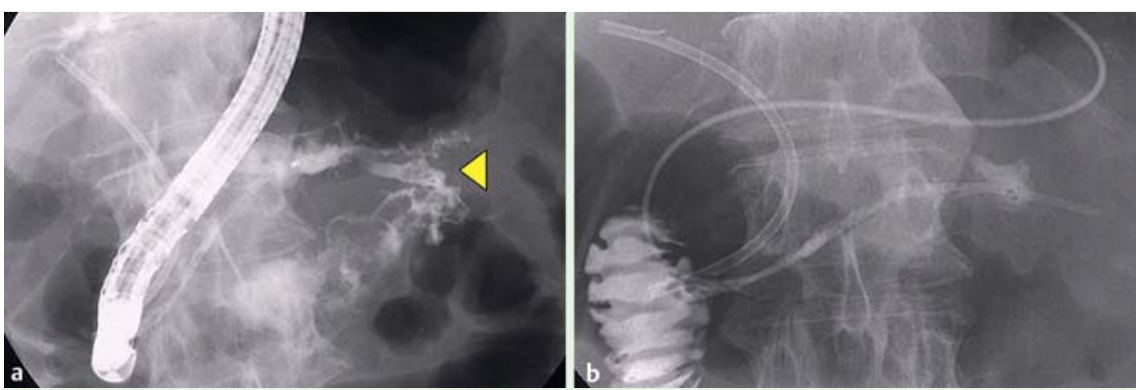

Fig. 4 a Endoscopic retrograde pancreatography performed after 2 weeks showing a colonic fistula at the distal main pancreatic duct (MPD) (arrowhead). b Contrast-enhanced scan after 10 days showing no evidence of the fistula.

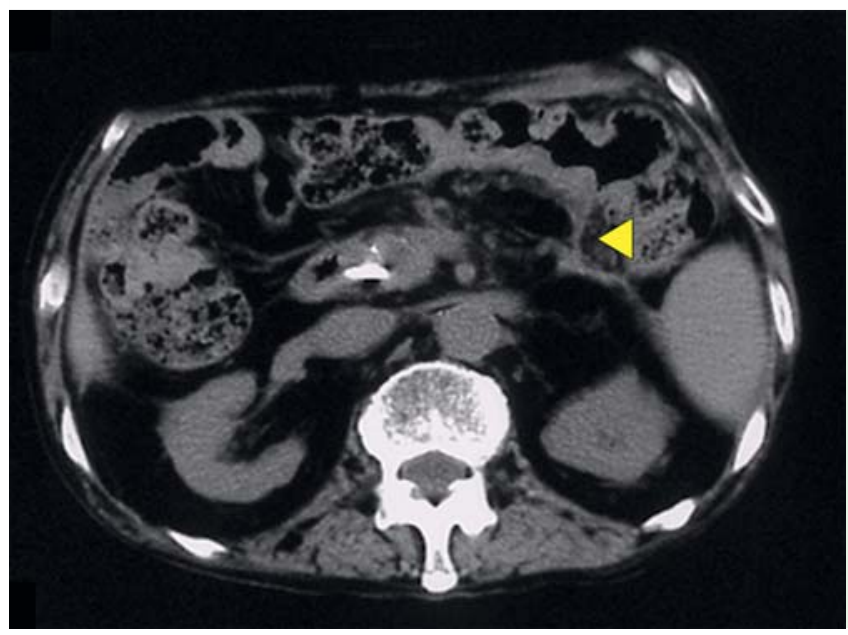

Fig. 5 Resolution of inflammation around the transverse colon with a fistula scar (arrowhead).

K. Fujii' ${ }^{1}$, K. Suzuki ${ }^{1}$, A. Goto ${ }^{1}$, K. Nakahata $^{1}$, Y. Matsunaga ${ }^{1}$, H. Wakasugi ${ }^{1}$, M. Itoh ${ }^{1}$, K. Yonezawa ${ }^{1}$, T. Abe ${ }^{1}$,

\section{Y. Shinomura²}

1 Department of Gastroenterology, Kushiro City General Hospital, Kushiro, Japan

2 First Department of Internal Medicine, Sapporo Medical University, Sapporo, Japan cases. Am J Gastroenterol 2000; 95: 1821 1823

4 Urakami A, Tsunoda T, Hayashi J et al. Spontaneous fistulization of a pancreatic pseudocyst into the colon and duodenum. Gastrointest Endosc 2002; 55: 949-951

5 Fukuda K, Izumi T, Nagase Y et al. A case of pancreatic abscess following acute pancreatitis with colonic fistula successfully treated by EUS-guided transgastric cyst drainage. Gastroenterol Endosc 2010; 52: 1275-1280

\section{Bibliography}

DOI $10.1055 / \mathrm{s}-0030-1256233$

Endoscopy 2011; 43: E154-E155

(c) Georg Thieme Verlag KG Stuttgart · New York . ISSN 0013-726X

\section{Corresponding author \\ K. Fujii, MD, PhD}

Department of Gastroenterology

Kushiro City General Hospital

1-12 Shunkodai

Kushiro 085-0822

Japan

Fax: +81-154-414080

kh8927@kushiro-cghp.jp 